\title{
Advanced Application of Flexible Metamaterials at Visible Frequencies
}

\author{
Blair C. Kirkpatrick, Peter Reader-Harris, Yufang Shen, Jingzhi Wu and Andrea Di Falco \\ University of St Andrews, Department of Physics and Astronomy, St Andrews, UK
}

\begin{abstract}
Flexible metamaterials (FMMs) at optical frequencies can conform to a wide range of target geometries whilst allowing their optical properties to be tuned post-fabrication. Here we discuss this potential by presenting our recent and preliminary results, obtained for FMMs at visible frequencies for sensing, filtering and imaging applications.
\end{abstract}

Keywords: Flexible Metamaterials, Lab on Fibre, Optomechanics, Thin Membranes

\section{INTRODUCTION}

Optical metamaterials (MMs) are materials made from sub-wavelength periodic structures, giving rise to optical properties not found in any natural material. This allows them to be used in many exotic applications, such as superlensing ${ }^{1}$ or cloaking. ${ }^{2}$ Historically MMs have been 3-dimensional, rigid structures, however, flexible metamaterials, operating at visible wavelengths, are now fully realisable. ${ }^{3}$ FMMs can be used to transfer a tailored photonic function onto existing devices or materials, thus decoupling the fabrication requirements from the geometry of the object itself, meaning that virtually any object can be dressed with a photonic response. In addition to this, their flexible nature also allows their optical properties to be tuned post-fabrication. In this paper we first present two different fabrication protocols and we then illustrate the advantage of this platform with specific applications in filtering, dispersion management and all-optical manipulation.

\section{FLEXIBLE METAMATERIALS}

FMMs can either be made of a one-dimensional stacks of layers of different materials or of a single patterned surface, typically metallic on a convenient substrate. Much of the basic fabrication procedure is the same for both classifications, although the latter fabrication steps vary in each case. Multilayer FMMs are typically made stacking dielectric and metal ultra thin layers, with maximum thickness much smaller than the wavelength. When this condition is satisfied the stack can be seen as homogenous by impinging light. For polarisation perpendicular to the stack, the effective permittivity, in the homogenised case, reads as $\epsilon_{e f f}=\left(\epsilon_{d} t_{d}+\epsilon_{m} t_{m}\right) /\left(t_{d}+t_{m}\right)$, where $\mathrm{t}$ is the layers' thickness and the subscripts $m$ and $d$ refer to the metal and the dielectric material, respectively. From this expression, it is clear that a judicious choose of parameters can lead to the condition $\epsilon_{e f f}=0$ (epsilon near

Further author information: (Send correspondence to A.D.F)

A.D.F.: E-mail: adf10@st-andrews.ac.uk, Telephone: 01334423165

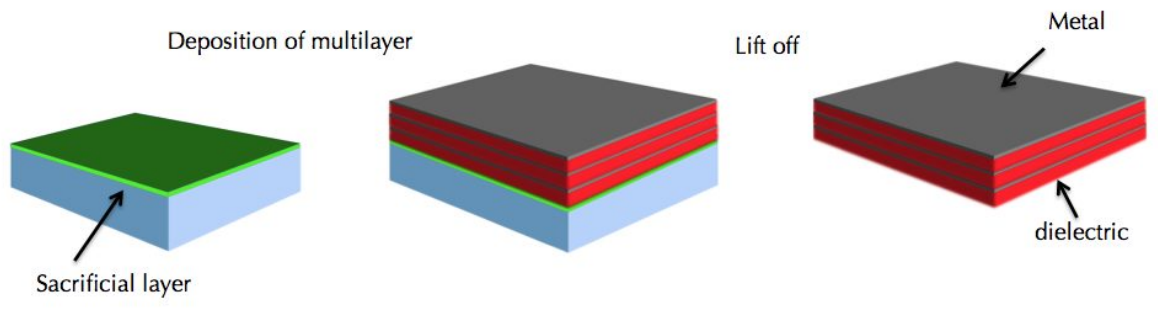

Figure 1. Standard fabrication protocol of a flexible metamaterial made of a one dimensional stack.

Photonic and Phononic Properties of Engineered Nanostructures V, edited by Ali Adibi,

Shawn-Yu Lin, Axel Scherer, Proc. of SPIE Vol. 9371, 93710Z - (c) 2015 SPIE

CCC code: $0277-786 X / 15 / \$ 18 \cdot$ doi: $10.1117 / 12.2078904$

Proc. of SPIE Vol. $937193710 Z-1$ 

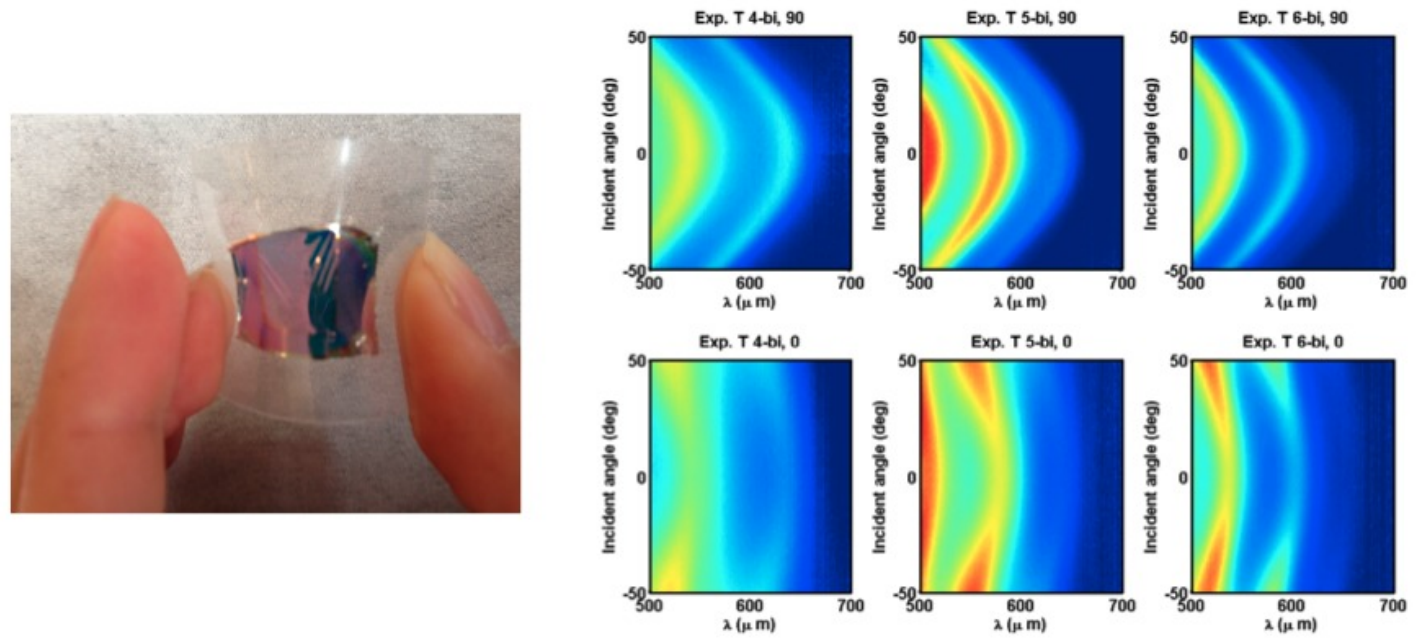

Figure 2. Left: a flexible multilayer epsilon near (ENZ) sample, mounted on a plastic frame. Right: Transmission spectra for 4,5 and 6 bilayers samples for different angles and polarisations.

zero, ENZ), for a given wavelength, due to dispersion of the materials. ${ }^{4,5}$ The fabrication procedure is sketched in fig. 1, for the specific case in which SU8 (a popular negative epoxy-based photoresist from Microchem) is used as polymer. A suitable commercially available SU8 release layer (omincoat) is spun on a rigid carrier, typically silicon. A thin layer of SU8 is then spun on the sample. The choice of SU8 is motivated by the high range and accuracy of film thicknesses readily available. For this samples, the thickness was kept in the range of $80 \mathrm{~nm}$, hence 8-10 times smaller than the target wavelength (at the edge of of the visible range). The polymer is then cured at $100 \mathrm{C}$ for 1 minute, activated via flood exposure to UV light for a further minute and cross linked with a 2 min long post exposure bake at 100C. The metallic layers are deposited via electron beam evaporation at a rate below $0.3 \mathrm{~nm} / \mathrm{s}$. Before this step, It is important to treat the polymer, e.g. with oxygen plasma, to increase the wetting of the metals, now reduced due to the acidic surface, effect of the cross linking process. The deposition can be iterated as many times as required and the stack eventually released via immersion in a suitable solvent (e.g N-Methyl-2-pyrrolidone).

The left panel of fig. 2 show a typical released FMM sample mounted on a plastic frame for convenience. The right hand panels of the same figures show the measured transmission spectra for different angles and polarisation (the top panels refer to the polarisation parallel to the rotation axis and perpendicular to the stack), for 4,5 and 6 bi-layers. It should be noted that the ENZ condition does not change for different angles, as the ratio between the layers does not change. The ENZ condition can be verified measuring the reflection spectra and retrieving the effective permittivity, with a standard fitting technique. This result will be the subject of further investigations, also connected to the development of experimental realisation embedding gain media, aimed at limiting the effect of losses due to the metal layers. ${ }^{6}$

For patterned FMMs, a sacrificial polymer layer is first spin coated on top of the silicon substrate, before being spun again with SU-8. As before we evaporate a metal on top of this, here we use gold, which can then be patterned through a combination of electron beam lithography and reactive ion etching. Removal of the sacrificial layer results in the FMM detaching from the silicon substrate, leaving it free to be mounted onto a custom built frame. The flexible nature of FMMs makes them very well suited to lab-on-fibre (LOF) applications. ${ }^{7}$ We demonstrate an angularly robust guided mode resonance (GMR) filter capable of being draped over the end facet of an optical fibre..$^{8,9}$ The filter's response originates from the interaction of the gold grating patterned on the FFM's surface (Fig. 3) with the optical modes supported the membrane itself. The advantage of the flexible substrate is that the given function of the GMR can effectively be transferred to the optical fibre without the need to pattern the fibre directly. ${ }^{10}$ This is especially true if the fiber is a hollow core, like in the care of the figure, thus not suitable for direct writing techniques. 


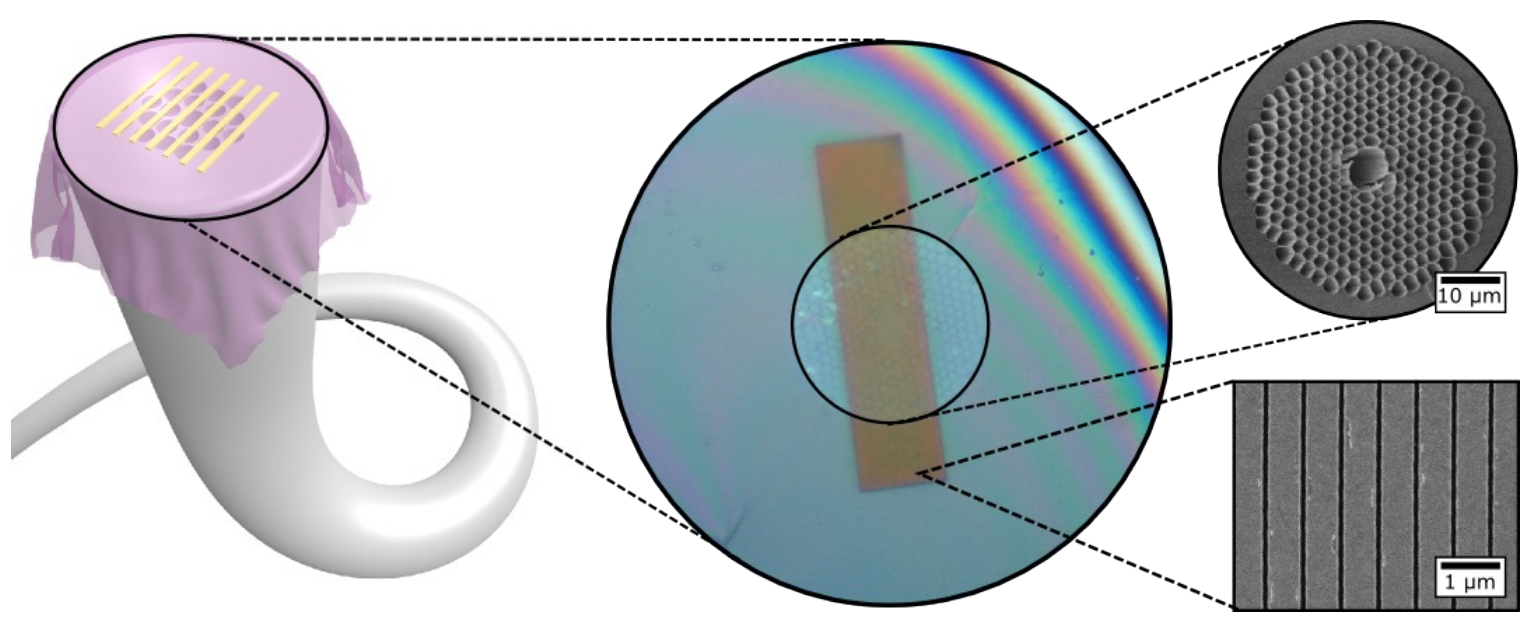

Figure 3. Image depicting (from left to right): the concept for FMM GMR filter draped across end fact of a photonic core optical fiber, microscope image of the end facet of such a fibre, and two images showing the structure of the photonic core and the GMR filter, both obtained via scanning electron microscope.

\section{OPTO-MECHANICS OF FLEXIBLE METAMATERIALS}

Not only can FMMs be utilised as a method to transfer photonic functionality to foreign objects, their flexibility also grants the possibility to modify a given photonic function simply by affecting the FMMs mechanically. This mechanical impetus can come in the form of a direct physical input or via a purely optical input. For the remainder of the discussion these will be referred to as "mechanical actuation" and "optical actuation", respectively. We investigate the opto-mechanical response of FMMs through both forms of actuation; mechanical actuation, in an air environment, through use of a piezo-electric crystal, and optical actuation, in a liquid environment, through the use of optical tweezers.

Fig. 4 shows a holographic reconstruction of the surface of a simple reflective membrane, supporting a standing wave pattern. By altering the driving frequency of a piezo actuator into which the membrane is mounted, it is possible to excite different standing mode patterns, thus enabling optomechanical experiments at the nanoscale. In order to utilise the functionality of FMMs in liquid, particularly for biological applications, a highly precise method for positioning them is required. To achieve this we use optical tweezers ${ }^{11}$ as they are capable of providing an unparalleled degree of control. Our fabrication procedure eliminates the need for nonnative "handles" to be added to our membranes ${ }^{12}$ via chemical processing. Instead, any features that are needed in order to increase trapping precision can be simply encapsulated in the design of the membrane itself, meaning no additional fabrication steps are needed (Fig. 5). Another protocol for manipulating FMMs in liquid is to utilise polymeric "microtweezers", which are fabricated using a near-identical method to the FMMs themselves. A release layer is spun onto a silicon substrate, with SU-8 polymer then being spun on top of that. This SU-8 is then patterned via electron beam lithography, creating the characteristic "tweezer" shape of the microtweezers (Fig. ??). From here they are then lifted-off, leaving them freely suspended in liquid, alongside the FMMs. The microtweezers can then be trapped using the optical tweezers and are capable of being opened and closed while they are positioned. By using these microtweezers to interact with and position the FMMs, we can limit the interaction between the FMM and the trapping laser light. Not only does this prevent the laser light from interfering with the function of the FMM, but the FMM is also protected from any potential damage caused by extended laser exposure.

\section{CONCLUSION}

FMMs extend the unique characteristics of metamaterials to a robust and highly flexible substrate. This allows a chosen functionality to be transferred to an object which, in general, can have a near-arbitrary geometry. Not only does this extend the regime in which metamaterial properties can be utilised, the target object can receive many different functionalities simply by removing and exchanging the appropriate FMMs. This versatility is 


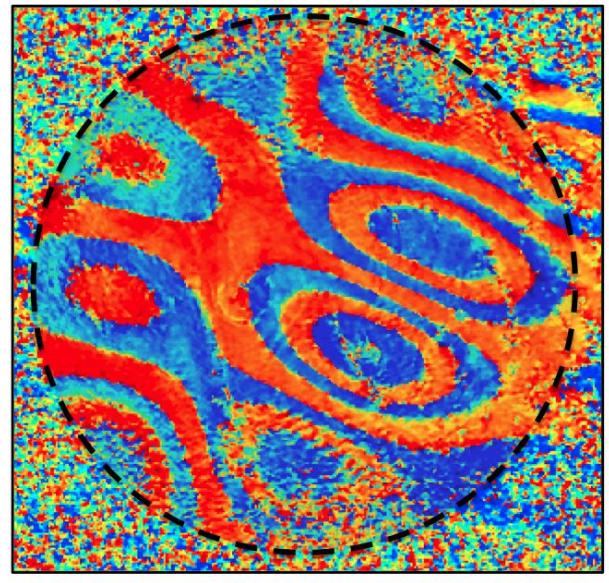

Figure 4. (a) Reconstructed hologram showing standing wave pattern on the surface of an oscillating membrane, actuated by piezo electric crystal. (b) Simulated bandstructure of Fabry-Perot oscillator with a FMM as it primary interface. The wavelength of light is plotted along the x-axis and the air gap of the Fabry-Perot is plotted along the y-axis. Intensity of the colour map relates to the strength of the reflected light, black representing zero reflection and white representing total refelection.
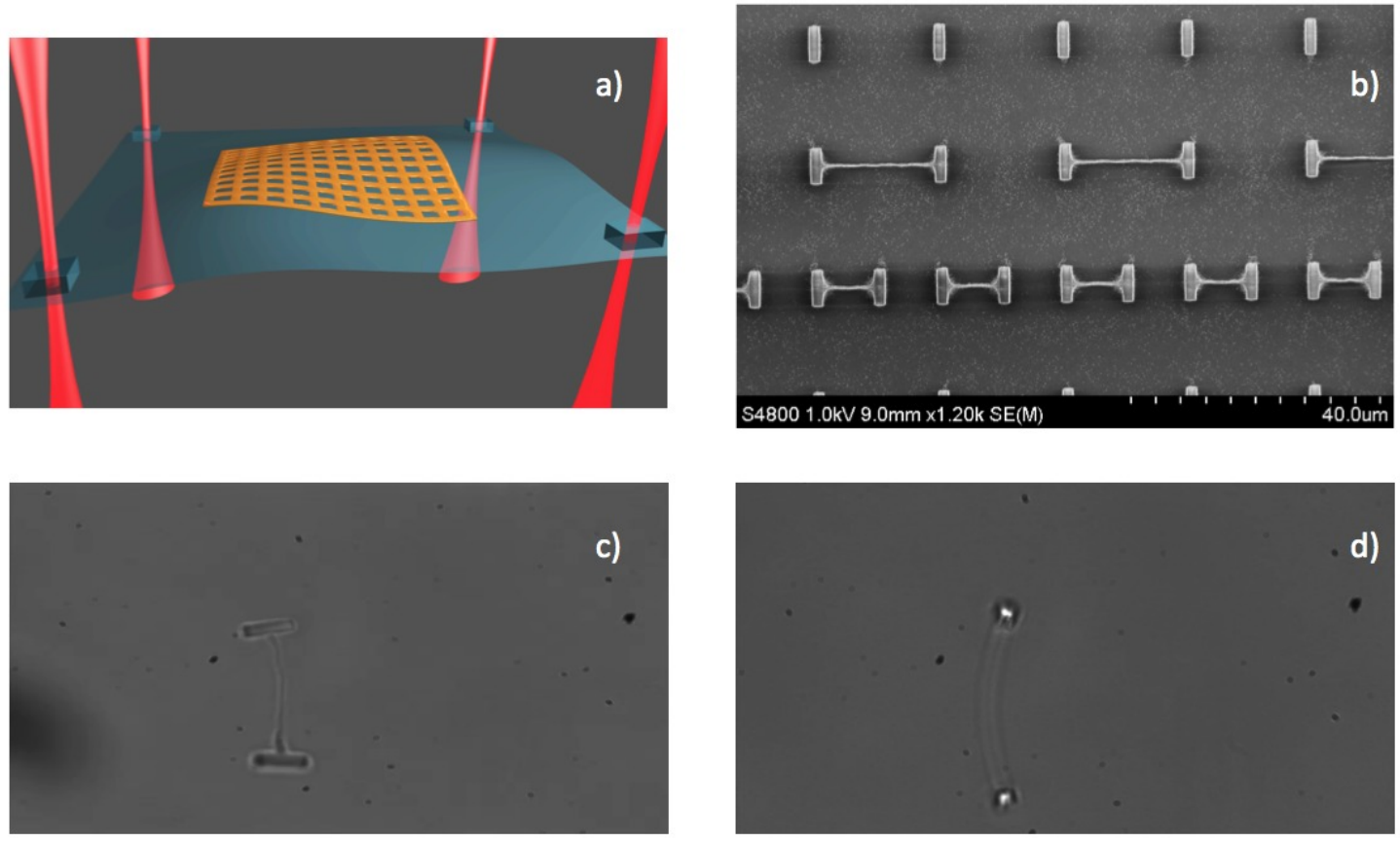

Figure 5. s a) Artist's impression of a FMM in liquid, held by four trapped polymeric handles. b) Image from a scanning electron microscope, depicting microscopic "tweezers", defined via electron beam lithography. The bottom panels show microscope images showing the tweezer structures suspended in liquid, in an optical trapping setup with traps off c) and on $(d)$. 
particularly evident in the case of flexible, reusable and angularly robust GMR filters. By tuning the electrical permittivity of multilayer FMMs, one can achieve an ENZ response at optical frequencies. These FMMs can ten be used as part of a super resolution setup, or as a test material for the study of nonlinear optical processes without the need for high optical powers. The structural flexibility of FMMs allows their properties to be tuned post-fabrication, allowing us to control the scattering properties of a FMM simply through mechanical actuation. This interplay between mechanical and optical properties is explored further with experiments in liquid. Using optical tweezers as the means of actuation, we investigate the potential biophysics applications of FMMs.

\section{ACKNOWLEDGMENTS}

We acknowledge support by EPSRC (EP/I004602/1 and EP/J004200/1).

\section{REFERENCES}

[1] Pendry, J., "Negative refraction make a perfect lens," Physical Review Letters 85, 3966 (2000).

[2] Schittny, R., Kadic, M., Bückman, T., and Wegener, M., "Invisibility cloaking in a diffusive light scattering medium," Science 345, 427 (2014).

[3] Di Falco, A., Ploschner, M., and Krauss, T., "Flexible metamaterials at visible wavelengths," New Journal of Physics 12, 113006 (2010).

[4] Alu, A., Silveirinha, M. G., Salandrino, A., and Engheta, N., "Epsilon-near-zero metamaterials and electromagnetic sources: Tailoring the radiation phase pattern.", Physical Review B 75, 155410 (2007).

[5] Ziolkowski, R., "Propagation in and scattering from a matched metamaterial having a zero index of refraction," Physical Review E 70, 046608 (2004).

[6] Rizza, C., Di Falco, A. and Ciattoni, A., "Gain assisted nanocomposite multilayers with near zero permittivity modulus at visible frequencies", Applied Physics Letters 99, 221107 (2011).

[7] Di Falco, A., Zhao, Y., and Alú, A., "Optical metasurfaces with robust angular response on flexible substrates," Applied Physics Letters 99, 163110 (2011).

[8] Reader-Harris, P., Ricciardi, A., Krauss, T., and Di Falco, A., "Optical guided mode resonance filter on a flexible substrate," Optics Express 21, 1002 (2013).

[9] Reader-Harris, P. and Di Falco, A. "Nanoplasmonic Filters for Hollow Core Photonic Crystal Fibers", ACS Photonics 1, 985 (2014).

[10] Lipomi, D., Martinez, R., Kats, M., Kang, S., Kim, P., Aizenberg, J., Capasso, F., and Whitesides, G., "Patterning the tips of optical fibers with metallic nanostructures using nanoskiving," Nano Letters 11, 632 (2011).

[11] Ashkin, A., Dziedzic, J. M., Bjorkholm, J. E., and Chu, S., "Observation of a single-beam force optical trap for dielectric particles," Optics Letters 11, 288 (1986).

[12] Oehrlein, S., Sanchez-Perez, J., Jacobson, R., Flack, R., Kershner, R., and Lagally, M., "Translation and manipulation of silicon nanomembranes using holographic optical tweezers," Nanoscale Research Letters 6 507 (2011). 